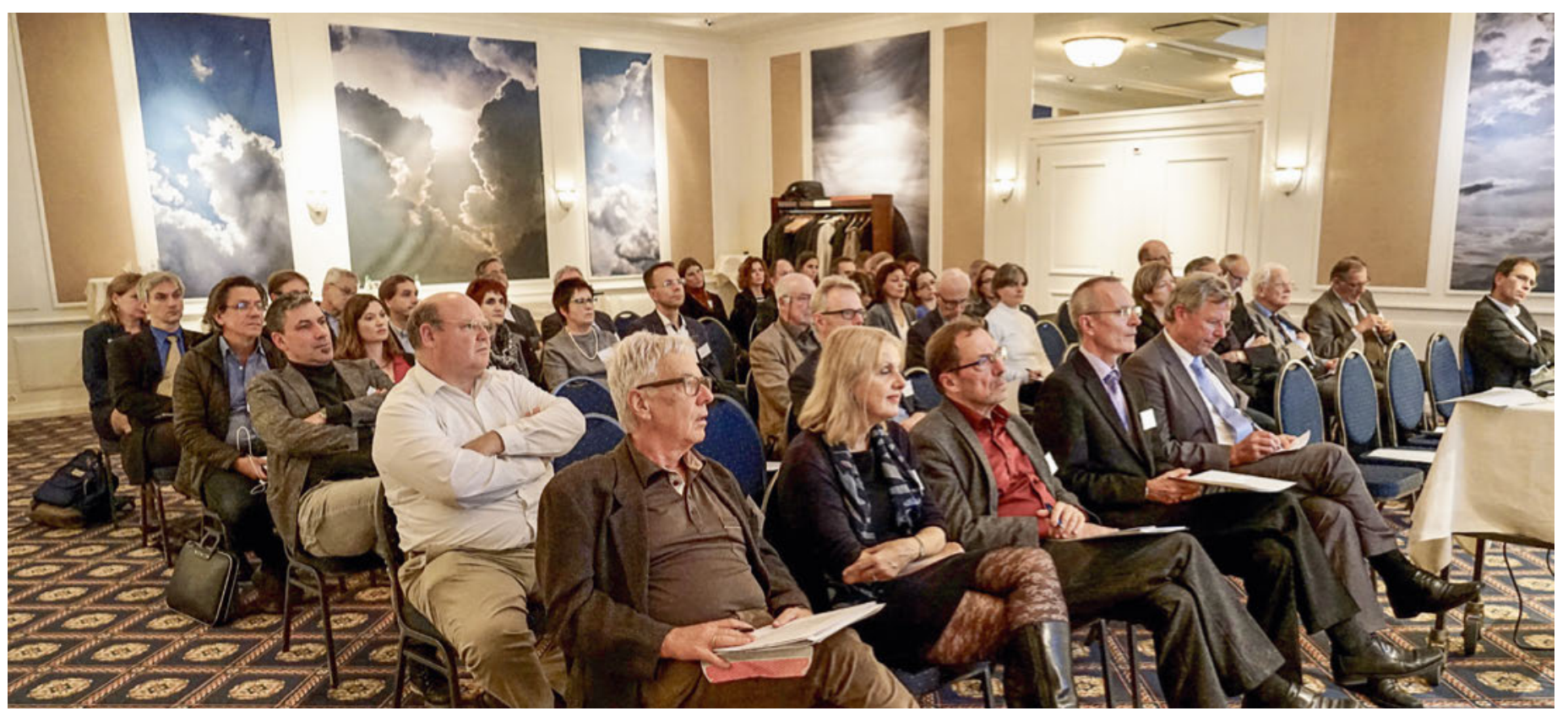

\title{
Des nuages sombres et du ciel bleu
}

\section{Matthias Scholer}

Rédacteur Bulletin des médecins suisses

Quelles sources d'information utilisez-vous dans votre activité professionnelle? Lisez-vous régulièrement des revues médicales? Plutôt imprimées ou en ligne? Une récente enquête gsf répond à ces questions et à bien d'autres. Outre le $20^{\mathrm{e}}$ anniversaire des Editions Médicales Suisses, les résultats de ce sondage étaient cette année le principal enjeu de la réunion des commissions de rédaction, qui a réuni de nombreux délégués de sociétés médicales.

«A partir de 2019, nous ne percevrons plus d'abonnement pour le BMS, le SMF et le SMW», a indiqué Ruedi Bienz dans son allocution de bienvenue, lors de la réunion des commissions de rédaction de cette année. Une date importante et peut-être un tournant pour les Editions Médicales Suisses EMH. Le Bulletin des médecins suisses (BMS), le Swiss Medical Forum (SMF) et le Swiss Medical Weekly (SMW) sont en effet les «produits phares» de la maison d'édition dirigée jusqu'à la fin de l'année par Ruedi Bienz. Celui-ci a également rappelé aux participants une autre date importante, un peu éclipsée par les considérations financières: le $20^{\mathrm{e}}$ anniversaire des éditions EMH. Elles ont été fondées en 1997 par la FMH, en collaboration avec la traditionnelle maison d'édition Schwabe, à Bâle, afin de professionnaliser la communication avec les membres de la FMH d'une part et de proposer aux médecins des publications indépendantes de qualité dans les domaines de la politique de santé, de la formation initiale, postgraduée et continue, mais aussi des sciences et de la recherche. 


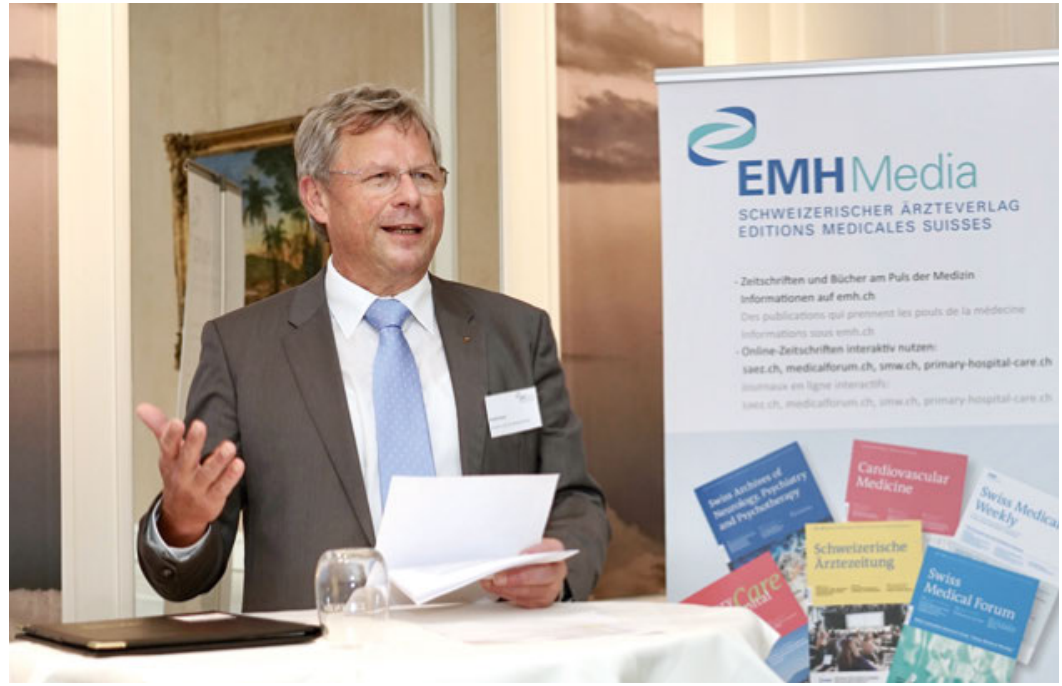

Ruedi Bienz, directeur EMH sortant.

Cet objectif a-t-il été atteint? C'est une question à laquelle les lecteurs sont seuls à pouvoir répondre. Comme le bureau d'études de marché et de recherche sociale gfs-zurich prévoyait de réitérer l'enquête «Presse médicale» réalisée en 2010, la FMH et les EMH en ont profité pour intégrer des questions ciblées sur leurs produits au catalogue de questions existant. Andreas Schaub, directeur de gfs-zurich, a pris le relais de Ruedi Bienz et commenté les principaux résultats et chiffres-clé de l'étude.

\section{Le système duel, clé du succès}

2550 médecins de diverses spécialités et de toutes les régions de Suisse ont participé à l'enquête, qui visait notamment à déterminer quelles revues médicales le groupe cible lit actuellement et comment il évalue leur qualité. Du point de vue des EMH, plusieurs résultats s'avèrent réjouissants et prometteurs. Les revues des EMH jouissent d'une très forte notoriété, sont beaucoup lues et très appréciées. De plus, l'indépendance rédactionnelle des revues spécialisées est un critère que les médecins jugent important à très important. Après la présentation des résultats du sondage, chaque rédacteur en chef a présenté les nouveautés de sa revue. Bruno Kesseli a profité de l'occasion pour rappeler le modèle duel du BMS aux personnes présentes. La première partie du Bulletin des médecins suisses est en effet le «journal officiel» de la FMH, ainsi que Kesseli l'a rappelé avec humour, tandis que la seconde est à la disposition du corps médical et des sociétés spécialisées en tant que plateforme d'information et d'échange encadrée par la rédaction.

Ce concept de contenu satisfait les lecteurs, comme en attestent les résultats de l'enquête. Le mix thématique des articles, de même que le fait que la revue soit ouverte aux médecins et à d'autres cercles du milieu de la santé, plaît à la grande majorité des sondés. La figure 2 présente les rubriques les plus appréciées des lecteurs. Bruno Kesseli a souligné que l'offre d'information du
1 Les résultats détaillés de l'étude sont disponibles en allemand sur le site Web emh.ch/fr/emh/ enquete-lecteurs.

\section{Globalement, quelle importance attachez-vous à l'indépendance} rédactionnelle des revues médicales?

Moyennes, échelle : $1=$ aucune importance à $5=$ grande importance, n $2010=$ env. $2800, \mathrm{n}$ total $2017=2140$, 2010: pondération par secteur d'activité et région, 2017: pondération par titre de spécialiste et activité principale

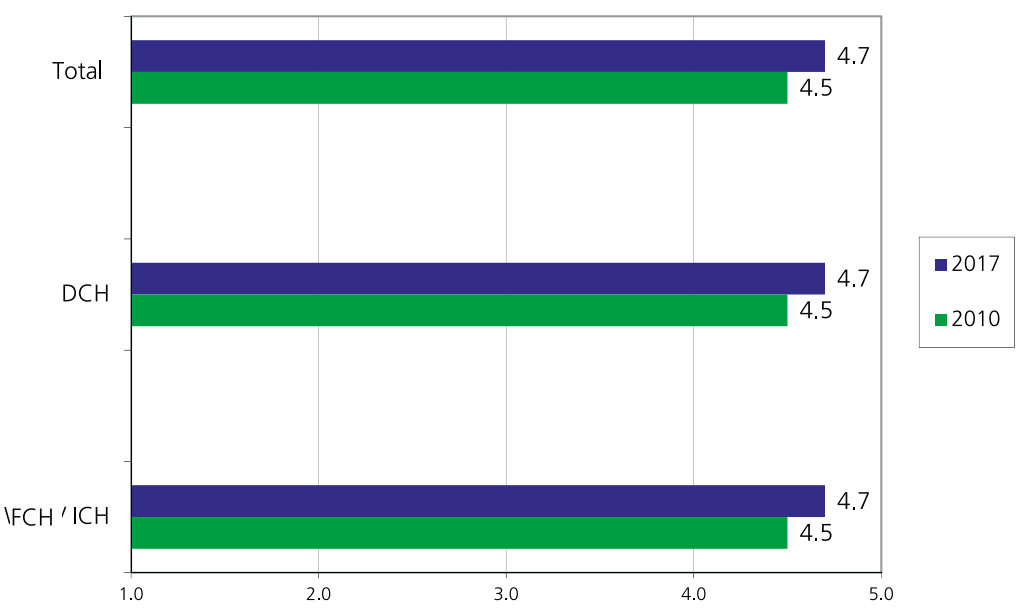

Figure 1: L'indépendance rédactionnelle, un critère important. 


\section{Bulletin des médecins suisses Comment lisez-vous les différentes rubriques? Tous les médecins}

Indications en pourcentages, échelle: de jamais à toujours, $n$ total $2017=2124$, pondération par titre de spécialiste et activité principale, filtre: a eu le BMS au moins une fois en main au cours des six derniers mois pour le feuilleter ou le lire (question au lectorat le plus vaste)

$$
\text { L'éditorial au début de la revue }
$$

Les informations sur la politique professionnelle/de la FMH Le courrier des lecteurs Articles d'opinion/points de vue Reportages, interviews, articles sur le thème clé Articles des pages culturelles du BMS (art, littérature, cinéma, histoire, expositions, critiques de livres) La chronique rédactionnelle «Et encore» La B.D. du BMS

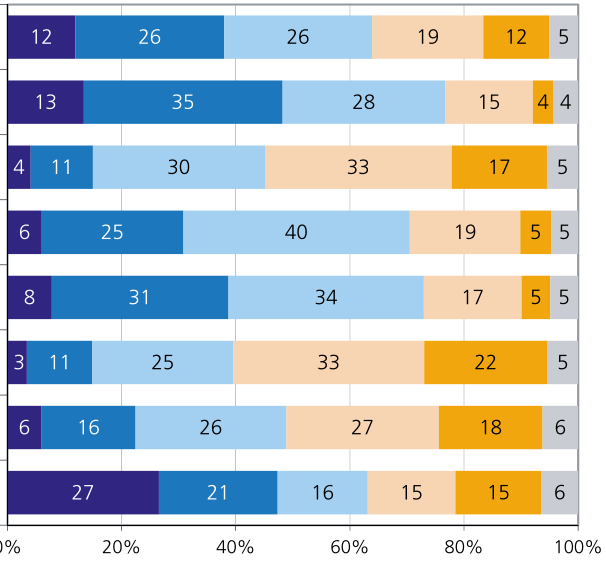

- Toujours $\backsim$ La plupart du temps $₫$ De temps en temps $₫$ Plutôt rarement $\backsim$ Jamais $₫$ ne sait pas/pas de réponse

Figure 2: Dans I'ensemble, le concept de contenu du BMS plaît.

BMS a l'an dernier été complétée de fonctionnalités en ligne modernes. La rubrique Web nouvellement créée «Tour d'horizon» permet par exemple un traitement rapide et multimédia de sujets de santé. La nouvelle page Facebook du BMS, qui n'existe pour l'instant qu'en allemand, doit également être vue comme un service complémentaire. La mise en ligne de la version française est prévue pour le début de l'année prochaine.

\section{De grands défis attendent les EMH}

En conclusion, Hans Kurt, le président du Conseil d'administration des éditions EMH, a résumé les propos du jour. Il s'est servi des motifs de nuages ornant les murs de la salle des fêtes pour illustrer la situation actuelle, comparant les grands défis auxquels les éditions EMH seront confrontées une fois les recettes d'abonne- ments supprimées à de sombres nuages d'orages. L'indépendance rédactionnelle et la qualité élevée des revues EMH pourront-elles être préservées dans ces conditions?

Entre les nuages, le décor mural comportait aussi des coins de ciel bleu, qui symbolisaient selon Hans Kurt la confiance dans la capacité des produits EMH à se maintenir en tant que source d'information importante des médecins dans leur quotidien professionnel. Les différents médias spécialisés s'appuient en effet sur une équipe de rédaction engagée et un personnel d'édition professionnel, sans lesquels le BMS, le SMF et le SMW ne seraient pas ce qu'ils sont.

\section{Crédit photos}

Figures: gfs-zurich

Photos: Matthias Scholer 